\title{
Test Systems for Voltage Stability Studies
}

\author{
T. Van Cutsem (Chair), M. Glavic (Secretary), W. Rosehart (Past chair), C. Canizares, M. Kanatas, L. Lima, \\ F. Milano, L. Papangelis, R. A. Ramos, J. A. dos Santos, B. Tamimi, G. Taranto, and C. Vournas
}

\begin{abstract}
This paper describes the two test systems for voltage stability studies set up by the IEEE PES Task Force on "Test Systems for Voltage Stability Analysis and Security Assessment" under the auspices of the Power System Stability Subcommittee of the Power System Dynamic Performance Committee. These systems are based on previous test systems, making them more representative of voltage stability constraints. A set of representative results are provided for both systems, with emphasis on dynamic simulation. They illustrate various aspects such as longterm dynamics, voltage security assessment, real-time detection, and corrective control of instabilities. The value for educators, researchers and practitioners are emphasized.
\end{abstract}

Index Terms-Long-term dynamics, voltage instability, load tap changers, overexcitation limiters, emergency control, dynamic security assessment, test systems.

\section{INTRODUCTION}

$\mathbf{V}$ OLTAGE instability is considered a major threat for secure operation in many power systems [1], [2], [3]. Limited growth in transmission expansion, power transfers over longer distances and decommissioning of generation plants are among the reasons for performing voltage stability analyses for long-term and operational planning and realtime operation. Furthermore, the design of dependable and secure System Integrity Protection Schemes (SIPS) to contain voltage instabilities requires to perform numerous dynamic simulations. The importance of these problems has attracted the attention of both researchers and practitioners for several decades, and many publications are available on these topics.

Test systems and their modeling play an important role in understanding the phenomena being studied, especially when investigating new solution schemes and comparing them with previously proposed approaches. The creation of the Task Force (TF) was motivated partly by the observation that a significant number of publications were resorting to existing test systems not truly limited by voltage instability. As a result, these systems exhibit low critical voltages, with the consequence that secure system operation would be limited by other phenomena well before such low voltages are reached.

The TF report [4] presents detailed models and provides all needed data to simulate two test systems:

1) The Nordic test system.

2) The Reliability and Voltage Stability (RVS) test system. These systems are long-term voltage stability constrained, with some scenarios evolving into system collapse when, for instance, generators go out of step under the effect of degraded grid voltages and field current limitations.

All authors were members of the IEEE Task Force on Test Systems for Voltage Stability Analysis and Security Assessment, Power System Stability Subcommittee, Power System Dynamic Performance Committee. E-mails: t.vancutsem@uliege.be, mevludin.glavic@uliege.be, vournas@power.ece.ntua.gr, leonardo.lima@ieee.org
TABLE I

SUMMARY OF MAIN SYSTEM CHARACTERISTICS

\begin{tabular}{|c|c|c|}
\hline & Nordic & RVS \\
\hline Nominal frequency (Hz) & 50 & 60 \\
No. of buses & 74 & 75 \\
No. of lines & 50 & 33 \\
No. of transformers & 52 & 56 \\
No. of generators & 19 & 32 \\
No. of synchronous condensers & 1 & 0 \\
No. of loads & 22 & 17 \\
No. of (switched) shunts & 11 & 2 \\
\hline Total generation (MW) & 11506 & 3200 \\
Total load (MW) & 11060 & 3135 \\
\hline
\end{tabular}

Both test systems are presented here together with the results of dynamic simulations for well identified disturbances. This enables the users to reproduce the presented scenarios and, thereby, validate the implementation of their models in various software tools. A subset of data can be used in static analyses, but the emphasis has been on dynamic simulations.

The rest of the paper is organized as follows: An overview of the main features of the models is given in Section II. The Nordic test system is presented in Section III, and a sample of representative dynamic simulation results is given in Section IV. A similar presentation is made for the RVS test system in Sections V and VI, respectively. Concluding remarks are presented in Section VII.

Note that this paper is not aimed at offering a tutorial on voltage stability, nor providing a comprehensive list of publications. Its purpose is to further draw the Community's attention on the test systems set up by the $\mathrm{TF}$, and promote their use.

\section{MAin FEATURES OF THE MODELS}

The main characteristics of both test systems are summarized in Table I. The focus of the TF was on long-term voltage instability [5], although the test systems can exhibit other instabilities as demonstrated in one of the studies for the RVS test system (see Section VI-B3), and the models were chosen keeping in mind the minimal requirements for representing components and phenomena that play a significant role in this type of phenomenon. More precisely the models are those already involved in short-term dynamics (e.g. transient (angle) stability) studies complemented with an appropriate representation of:

- Load power restoration, mainly under the effect of automatic Load Tap Changers (LTCs) and/or thermostatic load control. LTCs have a limited range of available tap positions, and other restorative loads have also upper and 
lower limits. These limits are included in the system models and data.

- Over Excitation Limiters (OELs) acting after some delay to reduce the field currents of synchronous generators. OELs normally allow transient over-excitation (current above thermal limit) for a short time period to allow fault-on and post-fault excitation boost and thus enhance transient stability. The OEL has to bring the field current close to the rated value within the time required to avoid overheating as specified by IEEE/ANSI standard C50.132014 (and previous versions from 2005, 1989 and 1977) [1], [2], [6]. In the Nordic test system the OELs have fixed or inverse-time delay, and are of the takeover type, while in the RVS test system they have a stepwise approximate inverse time delay and are of the summed type.

- Discrete controls triggered by the voltage decline, such as automatic switching of shunt compensation, modified LTC control or undervoltage load shedding.

The data files are available for use by the following software packages:

- Industrial: PSS/e, DSA Tools, and PowerFactory.

- Academic: RAMSES, PSAT, ANATEM, and WPSTAB. and can be downloaded from: https://site.ieee.org/pespsdp/489-2/

\section{OVERVIEW OF THE NORDIC TEST SYSTEM}

\section{A. Overall Description and Instability Causes}

This test system is a significantly upgraded version of the former Nordic32 test system [7]. In particular, dynamic models and parameters are adjusted to make them more representative for voltage stability studies. It is a fictitious system, but bears similarities with the Swedish and Nordic system. This test system, very often with undisclosed modifications, has been used to study various facets of voltage instability (e.g. [8][10]).

The one-line diagram is given in Fig. 1. The system has rather long transmission lines with $400-\mathrm{kV}$ nominal voltage. Five lines are equipped with series compensation: 4031-4041 (two circuits) by $50 \%, 4032-4044$ by $37.5 \%, 4032-4042$ by $40 \%$, and $4021-4042$ by $40 \%$. The model also includes a representation of some regional systems operating at 220 and $130 \mathrm{kV}$, respectively. All 20 generators (19 synchronous generators and one condenser) are represented behind their step-up transformers, and all 22 loads at distribution level are controlled by the LTCs of the step-down transformers.

This system is made up of the following four areas:

- "North" with hydro generation and some load.

- "Central" with much higher load and thermal power generation.

- "Equiv" connected to the "North", which includes a simple equivalent of an external system.

- "South" with thermal generation, which is rather loosely connected to the rest of the system.

Table II gives the active power generated and consumed in each area.

All generators were modeled with three or four rotor windings, and saturation. Simple generic models were used for the

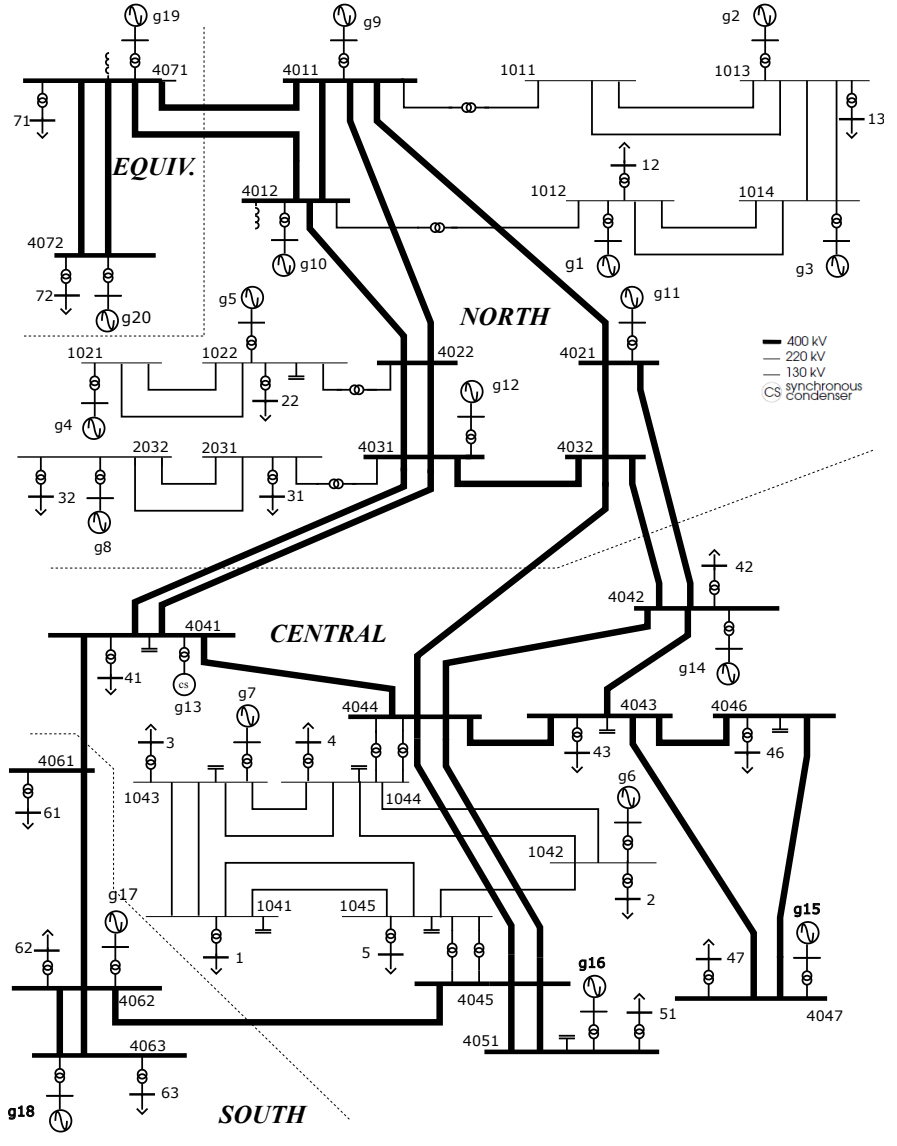

Fig. 1. One-line diagram of the Nordic test system.

TABLE II

LOAD AND GENERATION ACTIVE POWERS

\begin{tabular}{|c|c|c|}
\hline area & generation (MW) & load (MW) \\
\hline North & 4629 & 1180 \\
Central & 2850 & 6190 \\
South & 1590 & 1390 \\
Equiv & 2437 & 2300 \\
\hline
\end{tabular}

excitation systems, automatic voltage regulators, power system stabilizers, turbines and speed governors. With regarding to long-term dynamics the following modeling considerations were made:

- LTCs act with different delays on the first and subsequent tap changes, as well as from one transformer to another.

- The OELs of the four smallest generators act after a fixed time while all the others have inverse-time characteristics, i.e. the higher the field current, the faster its limitation.

All loads are connected to LTC-controlled distribution buses, and are modeled as constant current/admittance for the active/reactive power demand.

Frequency is controlled by the speed governors of the hydro generators in the North and Equiv areas only. The generator g20 is an equivalent generator, with a large participation in primary frequency control. The thermal units of the Central and South areas do not participate in this control. Generator $\mathrm{g} 13$ is a synchronous condenser.

The system is heavily loaded with large transfers essentially from the North to the Central areas. Secure system operation is 
limited by transient (angle) and long-term voltage instability. The contingencies likely to yield voltage instability are the following:

- The tripping of a line in the North-Central corridor, forcing the power to flow over the remaining lines.

- The outage of a generator located in the Central area, compensated by the Northern hydro generators, which adds to the power transfer in the North-Central corridor.

The maximum power that can be delivered to the Central loads is strongly influenced by the reactive power capabilities of the Central and some of the Northern generators; their reactive power is limited by OELs. On the other hand, the LTCs aim at restoring distribution voltages and hence load powers. If, after a disturbance (such as a generator or a line outage), the maximum power that can be delivered by the combined generation and transmission system is smaller than what the LTCs attempt to restore, voltage instability results. Thus, this is a long-term instability, driven by OELs and LTCs that takes place in a few minutes minutes after the initiating event. The instability mechanism is similar for demand increases.

\section{B. Operating Points}

Two operating points are considered. The first one, denoted A, is insecure, i.e. the system cannot stand some $\mathrm{N}-1$ contingencies. The system is made secure by rather simple modifications of operating point A leading to operating point B. The changes, depicted in Fig. 2, are as follows:

- In parallel with g16, an identical generator (g16b) with identical step-up transformer is connected, producing the same active power for the same terminal voltage. The additional production of $600 \mathrm{MW}$ is compensated by g20. The power flowing in the North-Central corridor is decreased by almost the same amount, which makes the system significantly more robust.

- For Operating Point B, the system could not withstand the loss of g15 or g18. Hence, these contingencies are made less severe by replacing each of these generators by two identical generators with half nominal apparent power, half nominal turbine power, and half power output.

\section{Simulation Results of Nordic test System}

\section{A. Response to a Large Disturbance}

In all presented cases, the disturbance is a three-phase solid fault on line 4032-4044, near bus 4032, lasting 5 cycles ( $0.1 \mathrm{~s}$ ) and cleared by opening the line, which remains opened. The evolution of two transmission bus voltages is shown Fig. 3 for initial operation at points $\mathrm{A}$ and $\mathrm{B}$, respectively.

For operating point $\mathrm{A}$, in response to the initial disturbance, the system undergoes electromechanical oscillations that die out in $20 \mathrm{~s}$. Then, the system settles at a short-term equilibrium, until the LTCs start acting at 35 s. Subsequently, the voltages evolve under the effect of LTCs and OELs. The system is long-term voltage unstable and eventually collapses, less than three minutes after the initiating line outage. As shown in the figure, essentially the Central area is affected;
Operating point $\mathrm{A}$
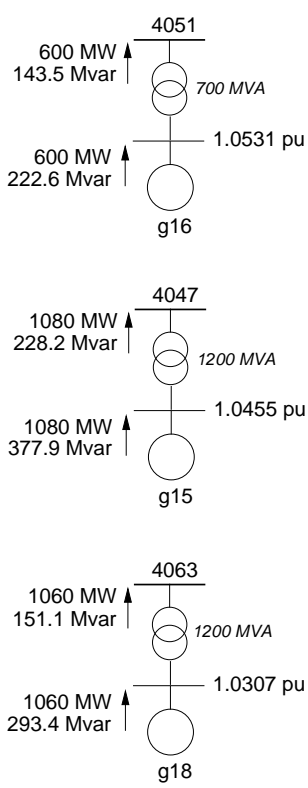

Operating point B
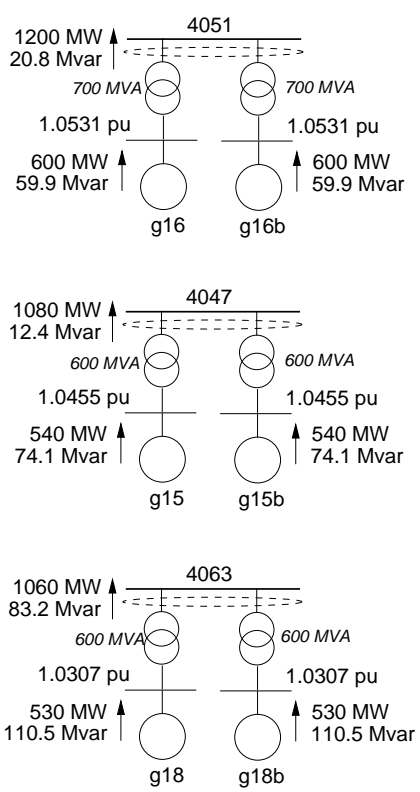

Fig. 2. Main differences between operating points A and B.

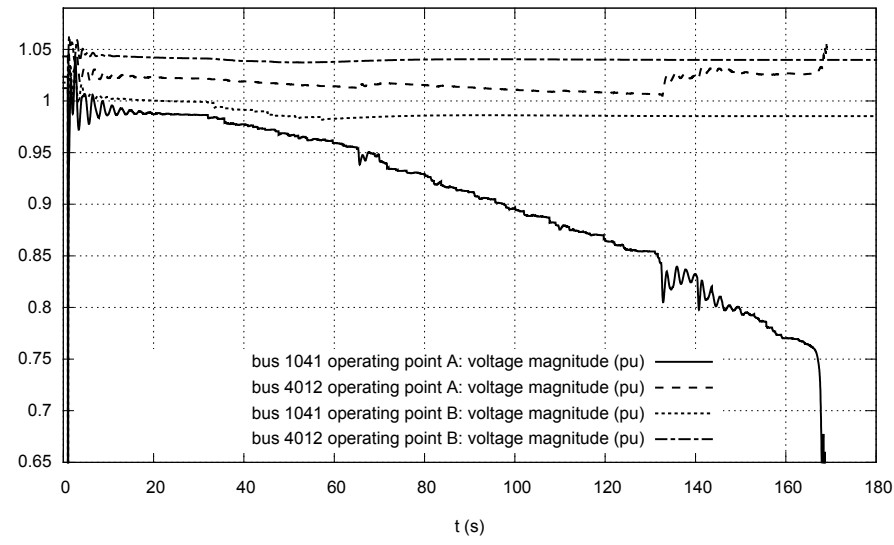

Fig. 3. Evolution of two transmission voltages at operating points A and B.

voltages in other areas are comparatively little influenced. The same figure shows the stable evolution of the same voltages when starting from operating point B.

Figure 4 shows the field currents of the seven generators that get limited for initial operating point A. Their OELs act in the order shown in the legend, from top to bottom.

The timing of OEL activation is illustrated in Fig. 5 for two of the seven generators. Under the effect of the fault, the field current of g11 exceeds transiently its limit, then comes back below it; thus, the OEL resets. The field current increases again after $t \simeq 35 \mathrm{~s}$ under the effects of LTCs and other generators getting limited. The field current limit is crossed again at $t \simeq 63 \mathrm{~s}$. As the OEL of g11 has a fixed activation delay of $20 \mathrm{~s}$, it reduces the field current at $t \simeq 83 \mathrm{~s}$. A similar evolution is observed for $\mathrm{g} 14$, but the field current settles above the limit sooner, at $t \simeq 3 \mathrm{~s}$. Unlike g11, the OEL of g14 obeys an inverse-time characteristic (the higher the overcurrent the 


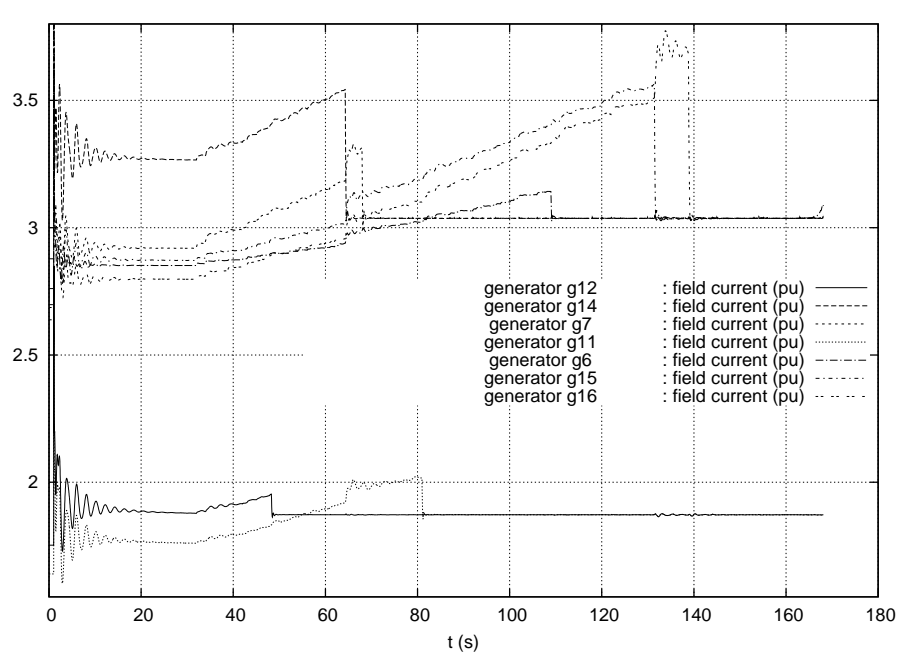

Fig. 4. Field currents of seven limited generators (oper. point A).

shorter the activation delay) and it acts at $t \simeq 67 \mathrm{~s}$.

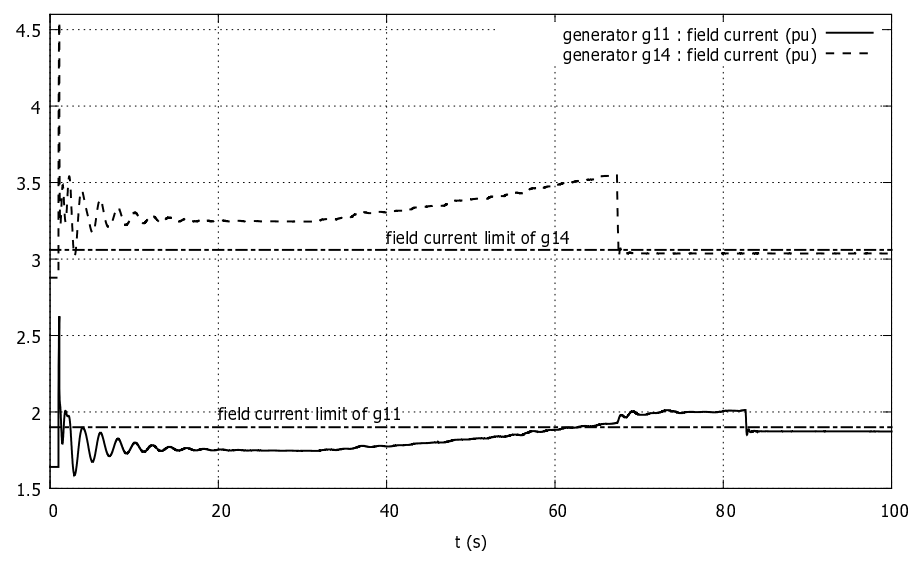

Fig. 5. Field current evolutions and corresponding limits of two generators.

Fig. 6 shows the evolution of the ratio of the transformer connected to bus 1041 and feeding the distribution bus 1, together with the voltage magnitude at that bus. The LTC fails to bring the distribution voltage back within the deadband (also shown in the figure). The distribution voltages, and hence the load powers cannot be restored to their pre-disturbance values, which is typical of long-term voltage instability. The figure also illustrates the multi-dimensional aspect of instability, i.e. interactions between LTCs. Indeed, each tap change brings a proper correction of the distribution voltage but, in between, the same voltage drops more under the effect of the other LTCs acting to restore their own voltages [8], [2].

\section{B. Determination of a Secure Operation Limit}

Voltage security is preventively assessed through the determination of a Secure Operating Limit (SOL), which involves stressing the system in its pre-contingency configuration. The stress considered here is an increase of loads in the Central area. The SOL corresponds to the maximum load power that can be accepted in the pre-contingency configuration such that

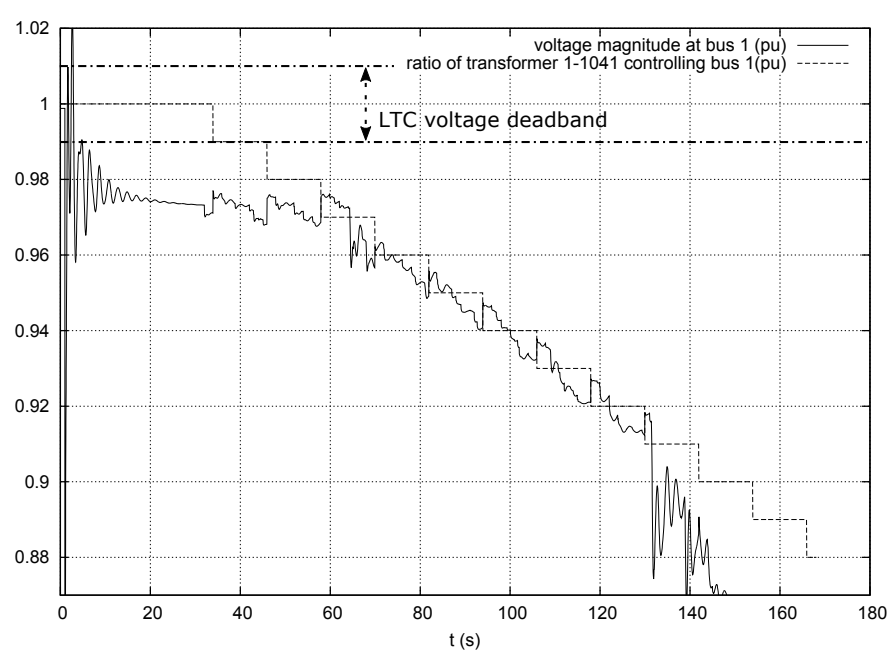

Fig. 6. Failed restoration of a distribution voltage by LTC (oper. point A).

the system responds in a stable way to each of the specified contingencies [2]. To this purpose, power flow computations were performed for increasing values of the Central active and reactive loads (in proportion to their base case value, and under constant power factor). For each so determined operating point, the disturbance is simulated. The system response is considered acceptable if, over a simulated time of $600 \mathrm{~s}$ : (i) all distribution voltages are restored in their deadbands; (ii) no generator voltage falls below $0.85 \mathrm{pu}$; and (iii) no loss of synchronism takes place.

In these pre-contingency power flow calculations, the active power variations are compensated by generator g20, while transformer ratios are adjusted as follows: the 22 distribution transformers are adjusted in order to maintain the distribution voltages in the deadbands, while the $400 / 130-\mathrm{kV}$ transformers 4044-1044 and 4045-1045 (see Fig. 1) are assumed to be controlled by operators, adjusting their ratio to maintain the voltages at buses 1044 and 1045 in specified ranges. The latter transformers do not have their tap changed in the postdisturbance simulation, whose duration is considered too short for operators to react.

Fig. 7 shows the post-disturbance evolution of the voltage at bus 1041, for various pre-contingency load levels. The case with $375 \mathrm{MW}$ loading seems stable but instability is revealed at about $800 \mathrm{~s}$. Thus, for that contingency, it is safe to set the SOL to $350 \mathrm{MW}$. Incidentally, the figure illustrates that, in marginal cases, it takes more time for the system to show its stability or instability.

\section{Corrective Control by a SIPS}

There are several possible control measures to restore stability. These include LTC blocking [3], tap reversing and voltage setpoint adjustment [11]. An example of SIPS corrective control is given in Fig. 8. The SIPS considered in this example consists of distributed undervoltage load shedding controllers as detailed in [12]. Each controller monitors the voltage at a transmission bus and acts on the load at the nearest distribution bus, according to the following simple rule: "curtail a step $\Delta P$ of load active power when the monitored transmission 


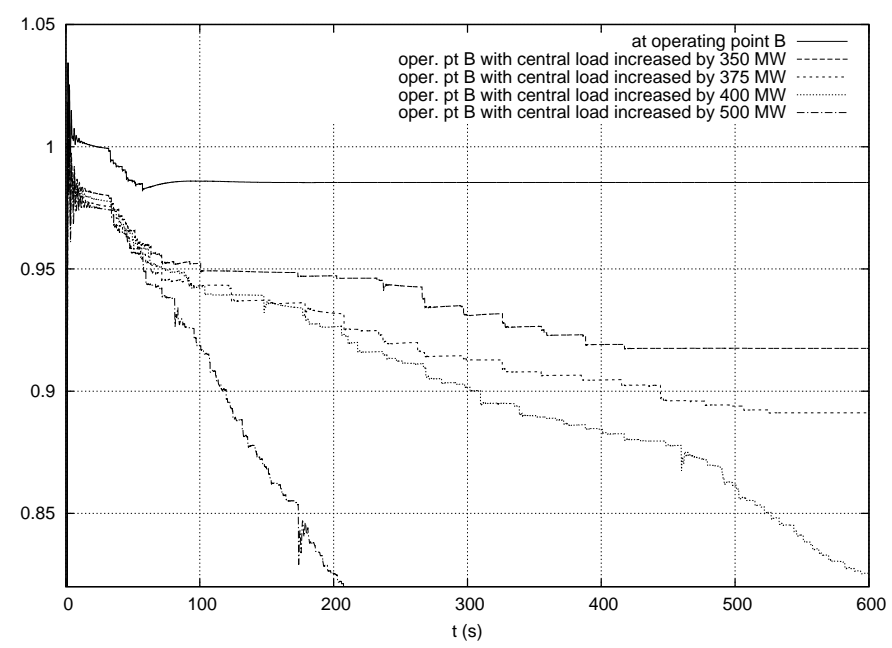

Fig. 7. Evolution of voltage at bus 1041 for various pre-contingency stresses.

voltage $V$ has stayed below a threshold $V^{t h}$ for more than a time $\tau$ ". Note that each controller can act several times, which yields a closed-loop behavior offering better robustness and adaptiveness. The various controllers do not exchange information but interact through the grid voltages.

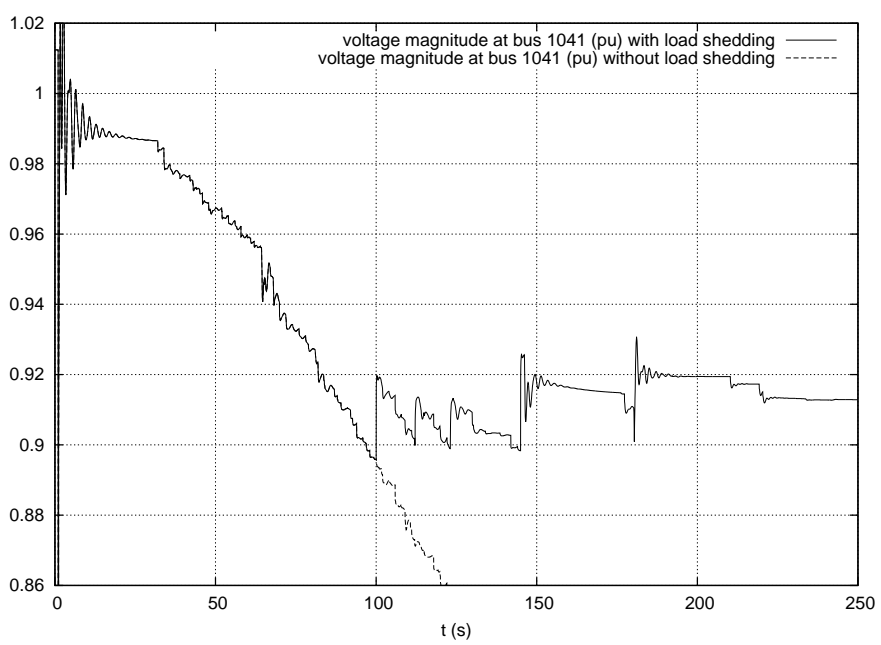

Fig. 8. Voltage magnitude at bus 1041, with and without load shedding.

The example in Fig. 8 was obtained with $V^{t h}=0.90$ pu, $\Delta P=50 \mathrm{MW}$, and $\tau=3 \mathrm{~s}$. The load reactive powers were decreased so that their power factors at $1 \mathrm{pu}$ voltage is preserved. A total of $300 \mathrm{MW}$ of load is shed in six steps: two by the controller monitoring bus 1041 and acting on bus 1 (at 100 and $144.95 \mathrm{~s}$ ), and four by the controller monitoring bus 1044 and acting on bus 4 (at 112.1, 123.1, 180.4, and $290.45 \mathrm{~s})$.

\section{PV Curve and Loadability Limit}

The last illustrative result deals with PV curves computed to determine the loadability limit of the system (without contingencies). In place of a continuation power flow, the PV curves were obtained by simulating a smooth ramp increase in demand, not far from what could be observed in a real system. The dynamic response was computed (with a time step size of $0.05 \mathrm{~s}$ ) with all components active, in particular LTCs and OELs. The simulation was stopped whenever a bus voltage reached an unacceptably low value, or it was ascertained that maximum load power had been crossed. PV curves are obtained by recording the evolutions of voltages and total load power with time, and plotting the former as a function of the latter.

The PV curves in Fig. 9 were obtained starting from operating point $\mathrm{B}$ and increasing all loads in the Central area (in proportion to their base case value, and under constant power factor), at a rate of $0.15 \mathrm{MW} / \mathrm{s}$ for the whole area. Primary frequency control was relied upon to compensate the power changes. To mimic manual control by operators, the 400/130-kV transformers 4044-1044 and 4045-1045 (see Fig. 1) were also equipped with LTCs adjusting the ratios in steps, with delays, to keep the voltages at buses 1044 and 1045 in pre-specified intervals.

The curves show a loadability limit near $6830 \mathrm{MW}$, yielding a load power margin of $640 \mathrm{MW}$. It can be seen that the voltage at bus 1044 is kept almost constant, owing to successive tap changes on transformers 4044-1044. The voltage at bus 1041 is not controlled by an LTC but that bus is electrically close to 1044; hence, its voltage drops a little during the load increase. On the other hand, at the transmission bus 4044, the decline is much more pronounced.

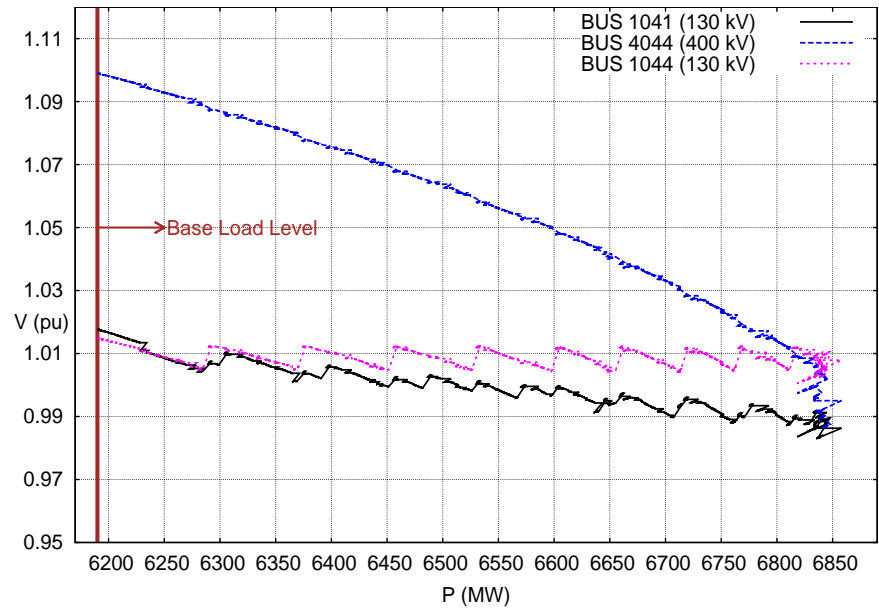

Fig. 9. PV curve for load increase in the Central area.

\section{OVERVIEW OF THE RVS Test System}

This test system corresponds to a large extent to the 1979 IEEE Reliability Test System [13]. The data presented in the TF report [4] relate to the so-called "one area RTS-96" system [14], which is equivalent to the 1979 Reliability Test System [13]. The one-line diagram of the network is shown in Fig. 10.

The original setting of these test systems or their modifications are used to study some aspects of voltage stability. References [15]-[18] present a sample of those works. The parameters of that benchmark system were adjusted to make voltage instability more pronounced. Several modifications 


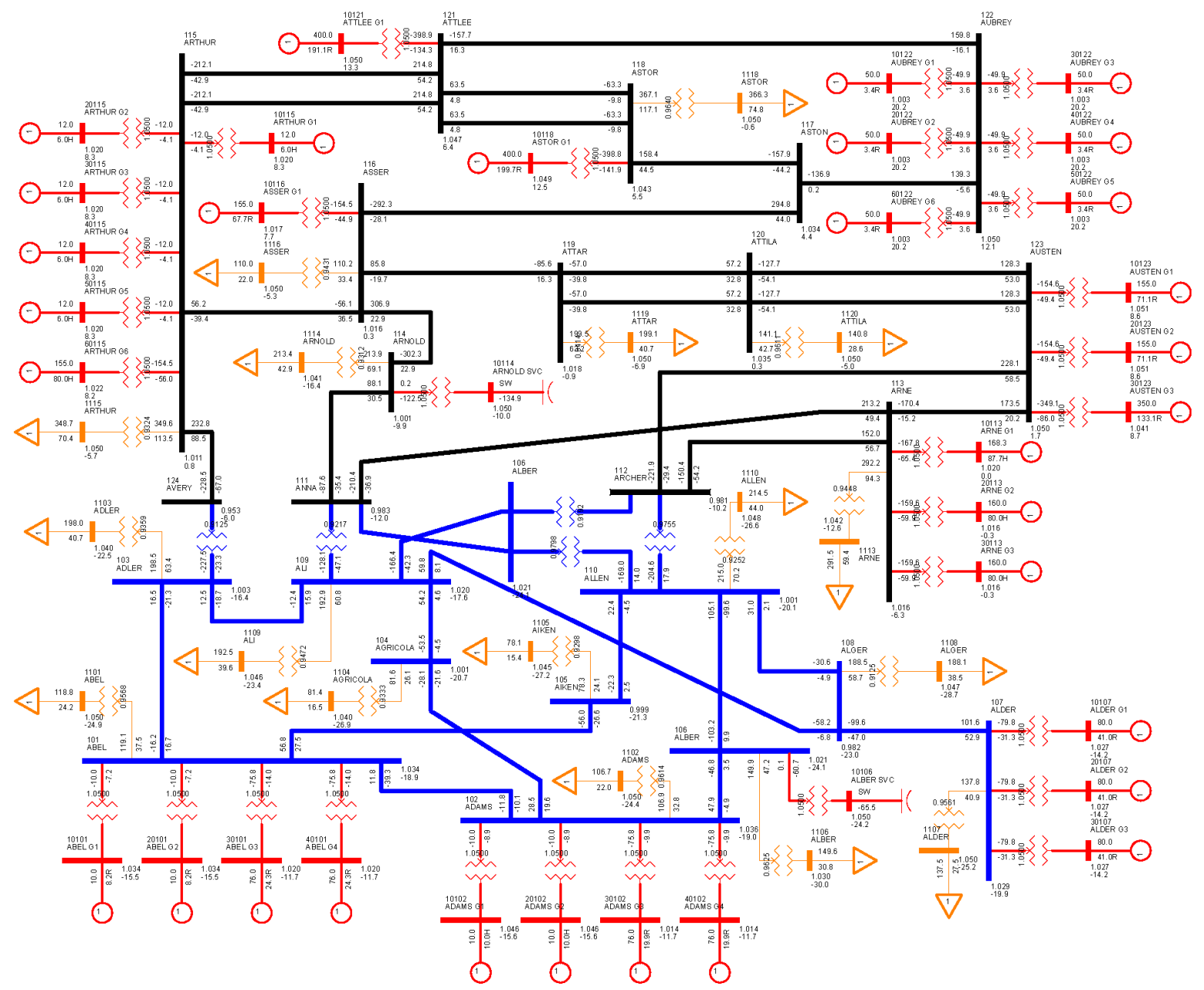

Fig. 10. One-line diagram of the RVS test system.

were also introduced in the power flow data of the original 1979 Reliability Test System, to make again the resulting model more suitable for voltage stability analysis. The main modifications were as follows:

- The synchronous condenser at bus 114 was replaced by a Static Var Compensator (SVC) with nominal range $-50 /+200$ Mvar. As is well known, the reactive power output of this device is voltage dependent and its reactive power output is significantly reduced under low voltage conditions.

- The shunt at bus 106 was replaced by another SVC with a range of $-50 /+100$ Mvar. This change introduces an additional degree of freedom that is very important for voltage control. This SVC is a key component in the RVS system and is required to avoid voltage instability. Usually the fast response of an SVC is not needed to improve long-term voltage stability. It is used in this system either with the understanding that it is equivalent to switched capacitor banks, or to improve voltage recovery after fault clearing in the presence of induction motors (see SectionVI-B3).

- The step-up transformers of generators and SVCs are explicitly represented, assuming five tap positions without LTC. The SVCs control their transmission-side voltages. The power flow solution considers all generators remotely controlling the voltage at the high-voltage side of their step-up transformers. In the dynamic simulation, all excitation systems control the generator terminal voltages.

- All other transformers are represented with LTCs allowing a $\pm 10 \%$ adjustment of the ratios in 33 steps $(0.625 \%$ per step). Each of them is located on the high-voltage side of the transformers and controls the voltage at the distribution (MV) side.

- Loads are no longer connected to the 138- or 230$\mathrm{kV}$ buses. Step-down transformers connecting LTCcontrolled $13.8-\mathrm{kV}$ buses were introduced with an estimated $0.15 \mathrm{pu}$ reactance on an MVA base set to the lowest multiple of 50 MVA greater than $110 \%$ of the load apparent power.

- The simulations were performed, initially, with all loads represented as $100 \%$ constant current for the active power and $100 \%$ constant impedance for the reactive power components.

- Power recovery and induction motor models were also included to study the impact of these types of loads in long- and short-term voltage stability phenomenon.

In this test system, OELs obey an inverse-time characteristic and act through the main summation point of the AVR. Thus, the OEL output signal is added to the voltage error signal, and can be thought of as a correction added to the voltage 


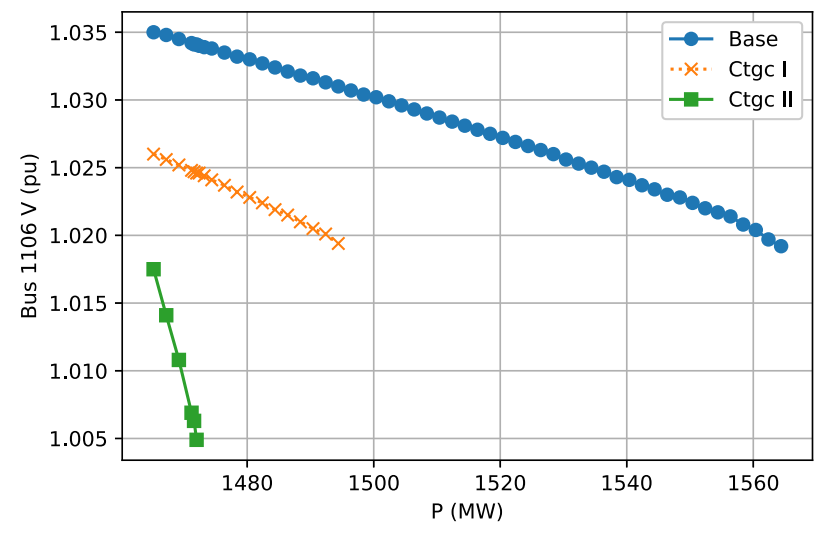

Fig. 11. PV curves for the base case and two contingencies.

reference setpoint. The model and associated parameters are documented in the TF Report. The inverse time characteristic is approximated by a piecewise linear curve defined by three pairs of points relating time and field current.

\section{Simulation RESUlts OF RVS TEST SYSTEM}

\section{A. Steady-state Analysis}

The steady-state analysis starts with the N-1 contingency analysis of all 230-kV and $138-\mathrm{kV}$ circuits. The following 3 contingencies led to non-convergent power flow conditions:

- Outage of the 230-kV circuit between buses 115 and 124 .

- Outage of the 138-kV circuit between buses 107 and 108 .

- Outage of the 138-kV circuit (cable) between buses 106 and 110.

Voltage stability of a system based on steady-state models is usually examined through its PV curves. The additional generation available at the generator buses to supply the load increase is close to $100 \mathrm{MW}$; thus, the power transfer increment in PV curve calculations is limited by this amount. The following two contingencies were also considered in PV curve calculations:

- Contingency I: Outage of the $230-\mathrm{kV}$ circuit between buses 111 and 114.

- Contingency II: Outage of the $230-\mathrm{kV}$ circuit between buses 112 and 123 .

The lines in these contingencies are important links for exchanging power between $230-\mathrm{kV}$ and $138-\mathrm{kV}$ subsystems. Moreover, for these contingencies, the system does not collapse and it is still possible to increase the load and study the system performance.

The PV curves obtained for the two contingencies and the base case are shown in Fig. 11. These plots correspond to the voltage at the load $(13.8-\mathrm{kV})$ bus 1106 . Note that Contingency II resulted in a maximum incremental transfer of just $10 \mathrm{MW}$.

\section{B. Dynamic Responses to Contingencies}

The following disturbances were considered in the dynamic simulations:

- Test I: Connecting a 250 Mvar reactor at bus 101 at $0.1 \mathrm{~s}$.

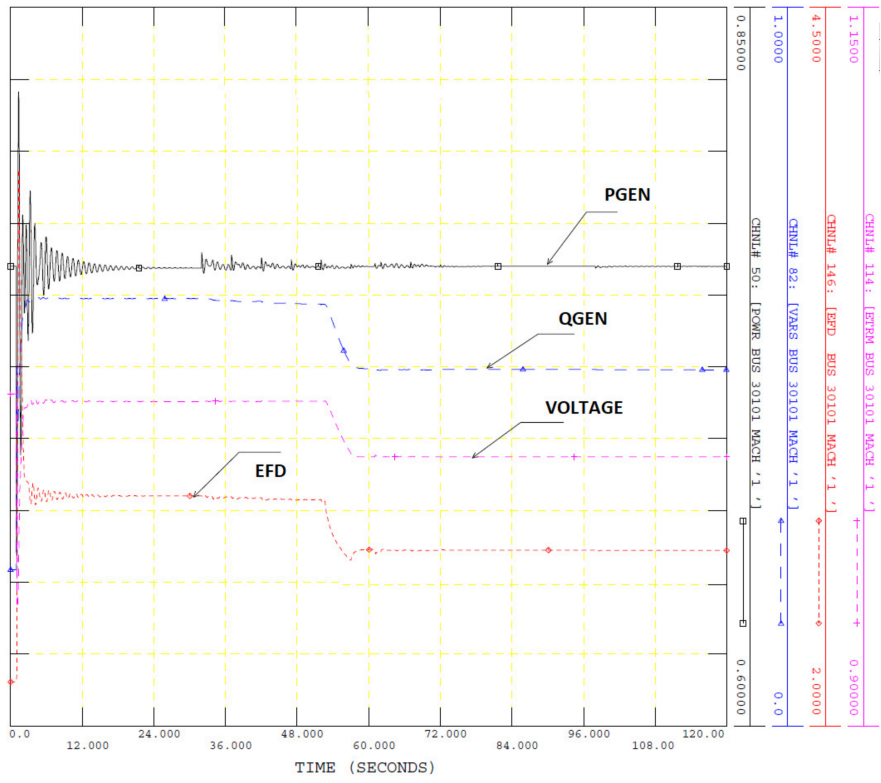

Fig. 12. Response of generator at bus 30101 (Test I).

- Test II: Outage of the cable between buses 106 and 110 without faults.

- Test III: Outage of the cable between buses 106 and 110 to clear a 3-phase fault at bus 106 after six cycles.

1) Test I: This test corresponds to a 250 Mvar shunt reactor being connected to bus 101 at $0.1 \mathrm{~s}$. Since this is a relatively small disturbance, the voltage at bus 101 drops from the initial value of $1.034 \mathrm{pu}$ to approximately $0.906 \mathrm{pu}$ once the reactor is switched on. This results in the two generators connected to bus 101 (generators 3 and 4 at buses 30101 and 40101) hitting their over-excitation limits.

Fig. 12 presents the response of the generator 3 (bus 30101). It shows the active and reactive power outputs of the machine (in pu of the system base, 100 MVA), terminal voltage, generator field voltage and the output of the OEL. Following the initial disturbance and after transients caused by the disturbance, the generator field current settles at $2.82 \mathrm{pu}$, higher than the rated field current for this machine $(2.473 \mathrm{pu})$, entering the inverse time characteristic of the OEL model. The OEL model becomes active at approximately $50 \mathrm{~s}$. Since the OEL model represents a summation point OEL action, the OEL starts to lower the voltage reference set-point for the generator, so that terminal voltage and reactive power output are ramped down until a new steady state is reached at lower voltages.

Fig. 13 shows the response of the load connected to bus 101 (13.8-kV bus 1101), where the effect of the on-load tap changers is clearly observed. The initial voltage at bus 1101 is $1.049 \mathrm{pu}$ and, after several steps in the LTC response, it recovers to $1.036 \mathrm{pu}$. Since voltage recovers to almost the initial value, the load demand recovers to close to its original power demand at the end of the simulation.

2) Test II: The second simulated disturbance corresponds to Figs. 14 to 16 which present the voltages at buses 106 and 1106, and the active power demand of the load at bus 1106 . It is known that aggregate loads, representing the combined 


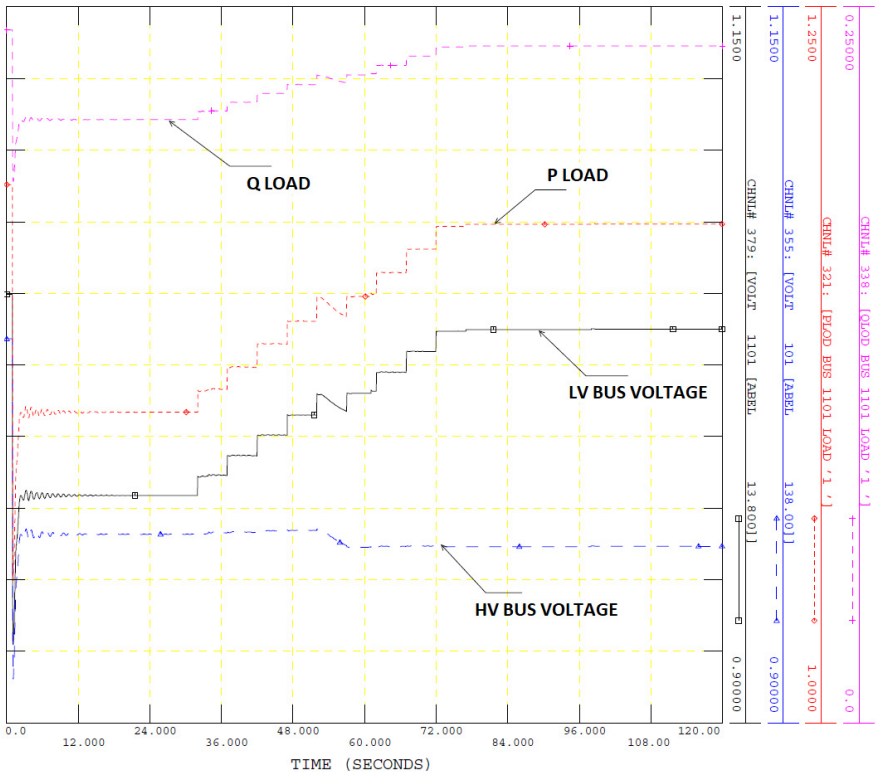

Fig. 13. Response of load at bus 1101 (Test I).

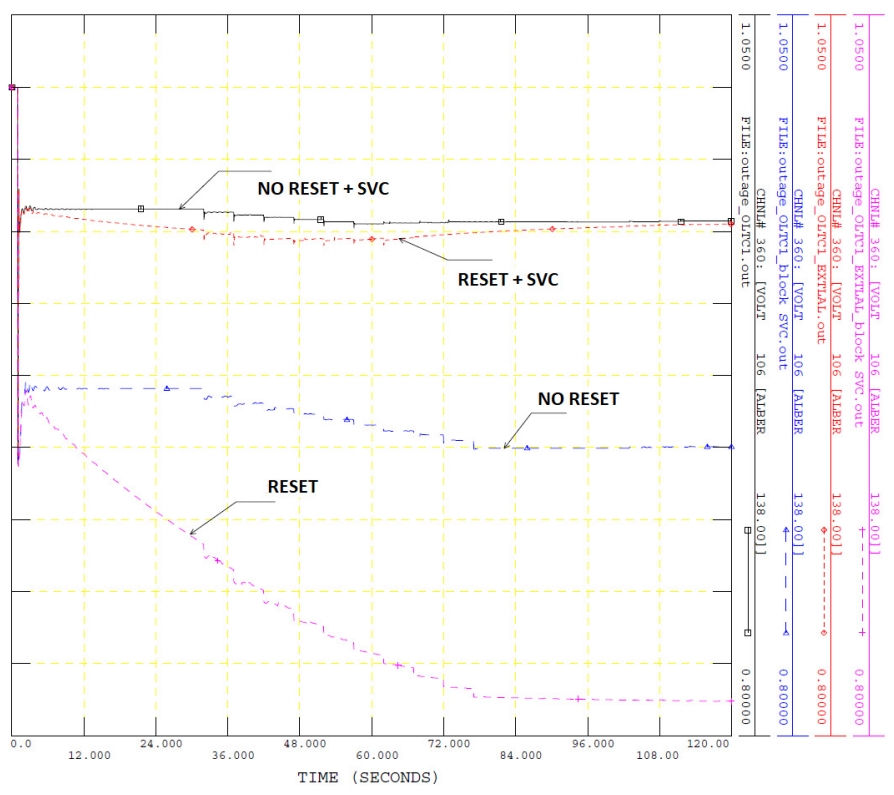

Fig. 14. Voltage at $138 \mathrm{kV}$ bus 106 (Test II).

demand from many consumers, tend to restore their power demand following a disturbance. Thus, it is necessary to represent the load recovery (referred to as RESET in the figures) in long-term dynamic simulations. The RESET model restores the pre-contingency load consumption, to show the effect of restorative loads, such as thermostatically controlled. However, the response rate is artificially increased to speed up simulation. Thus the RESET curves in the figures can be understood as lasting longer. Note that no interaction with other dynamics is introduced by this artificial speed-up.

It can be observed that the dynamic response of the SVC at bus 10106 is critical to avoid a voltage collapse condition around bus 106. In fact, the SVC response combines with the LTC response to bring the voltage at the load bus 1106

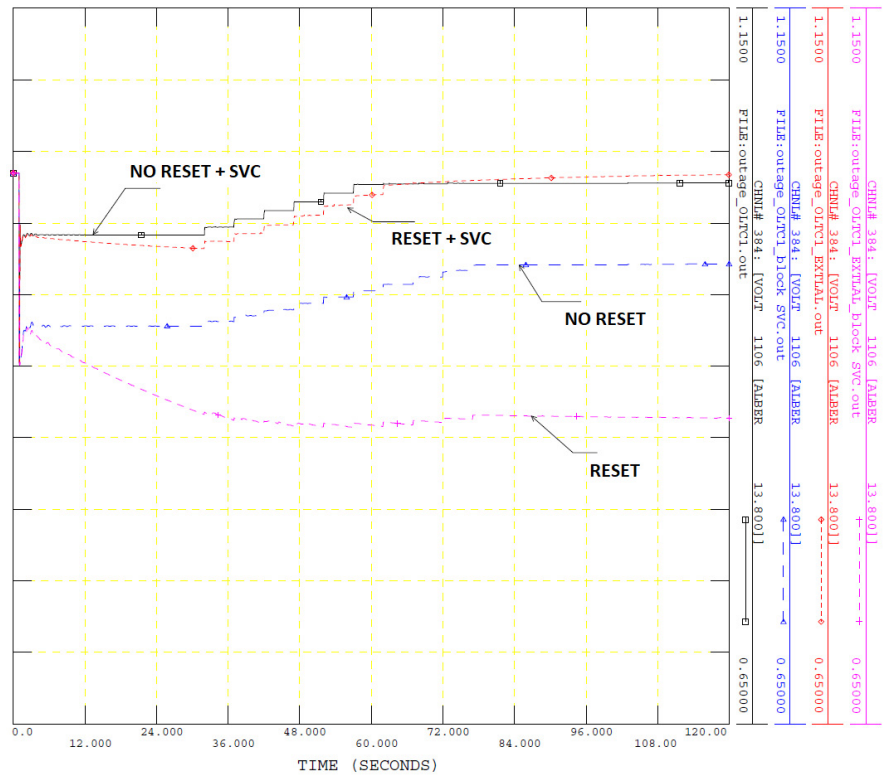

Fig. 15. Voltage at $13.8 \mathrm{kV}$ bus 1106 (Test II).

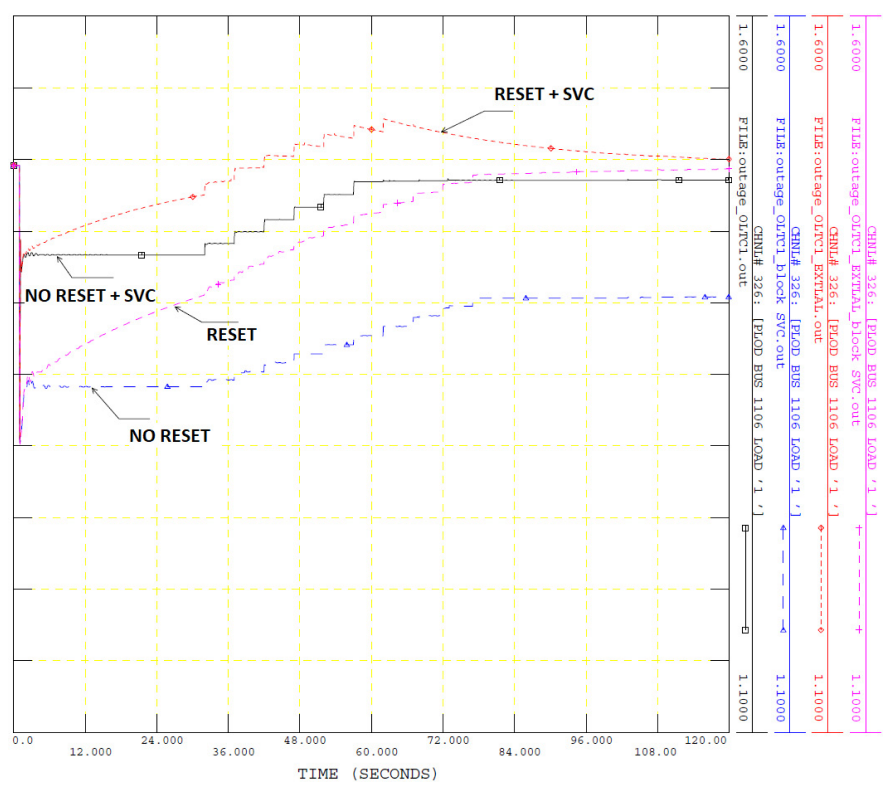

Fig. 16. Active power demand of load at bus 1106 (Test II).

to a higher value than the initial condition. Since the load is modeled with a voltage dependence characteristic, the load demand becomes greater than the initial value and the load recovery model ends up reducing the load demand.

On the other hand, when the SVC is blocked, the voltages at buses 106 and 1106 do not recover. Without the load recovery characteristic, these voltages stabilize at around 0.9 pu due to the associated reduction in active and reactive power demand. When the load recovers to its pre-disturbance power demand, voltages decrease even further and stabilize just above $0.8 \mathrm{pu}$.

Since this is a slow voltage collapse condition, it is conceivable that mechanically-switched capacitor banks could be used instead of SVCs. 


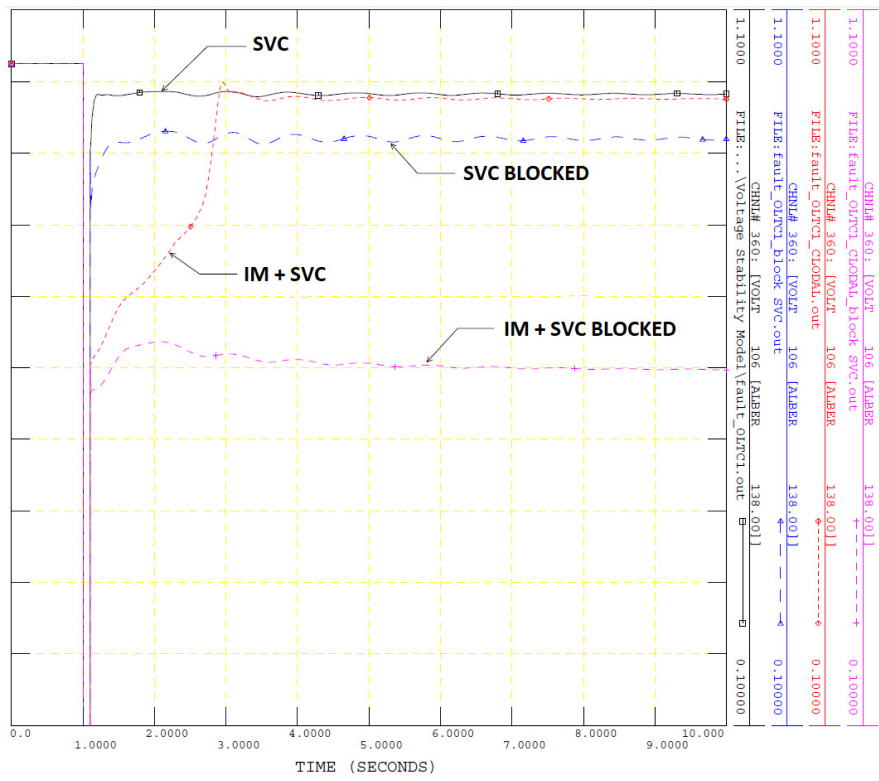

Fig. 17. Voltage at $138 \mathrm{kV}$ bus 106 (Test III).

3) Test III: The third simulated disturbance corresponds to Fig. 17, which present the voltage at bus 106 . Once again, the dynamic response of the SVC is critical to avoid a voltage collapse condition around bus 106, caused by the increase in reactive power demand due to stalling induction motors (IM). This is a fast dynamic phenomenon and, in this case, the control capability of the SVC is required to avoid sluggish voltage recovery and the possibility of load disconnection due to sustained low voltages. Further details can be found in [4].

\section{CONCLUSIONS}

The TF has provided case studies for education and research purposes, in particular to test new solutions for the assessment, detection and mitigation of voltage instability, and for relevant comparisons of methods and software packages. Although relevant, some components and controls were not considered by the TF, mainly because they are less widely used; armature current limiters, control of generator stepup transformer ratios, and secondary voltage control [3] are typical examples. These items could be considered in future extensions together with the models of other important components such as: alternative OEL models, HVDC links (of both LCC and VSC types), (converter-interfaced) generation dispersed in distribution networks, load dropout, and others.

Since the time of the TF completing its work, the test systems have been used in various research works (e.g. [11]). Furthermore, the models have been extended to cover other dynamic phenomena such as short-term voltage instability, delayed voltage recovery or frequency control. Examples of such studies are easily found on IEEEXplore and in the report of another IEEE TF [19]. Let us quote non exhaustively: replacement of a subset of synchronous generators by large-scale photo-voltaic systems for short- and long-term voltage studies, extension of the model into a combined transmission-distribution grid [19], low-frequency oscillations damping using battery energy storage, system reinforcement through point-to-point HVDC links, incorporation as AC area within a multi-terminal DC grid, addition of a communication infrastructure for cyber-physical security studies, frequency stability in the presence of large-scale wind farms.

The Power System Community is herein heartily encouraged to contribute to these updates by sharing the relevant documentation and data files. This update activity is being supported by the Working Group on Dynamic Security Assessment sponsored by the Power System Stability Subcommittee of the Power System Dynamic Performance Committee.

\section{REFERENCES}

[1] C. W. Taylor, Power System Voltage Stability, EPRI Power System Engineering Series, McGraw Hill, 1994.

[2] T. Van Cutsem, C. Vournas, Voltage stability of electric power systems, Springer (previously Kluwer Academic Publishers), Boston (USA), 1998.

[3] S. Corsi, Voltage Control and Protection in Electrical Power Systems, Springer, 2015

[4] T. Van Cutsem (Chair) et al., "Test systems for voltage stability analysis and security assessment," IEEE/PES Power System Stability Subcommittee, Tech. Rep. PES-TR19, Aug. 2015.

[5] C. Cañizares (Editor) et al. "Voltage stability assessment: concepts, practices and tools," IEEE/PES Power System Stability Subcommittee, Tech. Rep. PES-TR9, Aug. 2002.

[6] P. Kundur, Power system Stability and Control, EPRI Power System Engineering Series, McGraw Hill, 1994, pp. 337-339.

[7] M. Stubbe (Convener), Long-term dynamics - phase II, Report of CIGRE Task Force 38.02.08, Jan. 1995.

[8] C. Vournas, T. Van Cutsem, "Local identification of voltage emergency situations," IEEE Trans. on Power Systems, vol. 23, no 3, pp. 1239-248, Aug. 2008.

[9] M. Glavic, T. Van Cutsem, "Wide-area detection of voltage instability from synchronized phasor measurements. part I: principle. part II: simulation results," IEEE Trans. on Power Systems, vol. 24, no. 3, pp. 1408-1425, Aug. 2009.

[10] M. Glavic, M. Hajian, W. Rosehart, and T. Van Cutsem, "Recedinghorizon multi-step optimization to correct nonviable or unstable transmission voltages," IEEE Trans. on Power Systems, vol. 26, no. 3, pp. 1641-1650, Aug. 2011.

[11] C. D. Vournas, C. Lambrou, and M. Kanatas, "Application of local autonomous protection against voltage instability to IEEE test system," IEEE Trans. on Power Systems, vol. 31, no. 4, pp. 3300-3308, Jul. 2016.

[12] B. Otomega, M. Glavic, T. Van Cutsem, 'Distributed undervoltage load shedding," IEEE Trans. on Power Systems, vol. 22, no 4, pp. 2283-2284, Nov. 2007.

[13] Reliability Test System Task Force of the Application of Probability Methods Subcommittee, "IEEE reliability test system," IEEE Trans. on PAS, vol. 98, no. 6, pp. 2047-2054, Nov./Dec. 1979.

[14] Reliability Test System Task Force of the Application of Probability Methods Subcommittee, "The IEEE reliability test system-1996," IEEE Trans. on Power Systems, vol. 14, no. 3, pp. 1010-1020, Aug. 1999.

[15] R. Billinton, S. Aboreshaid, "Voltage stability considerations in composite power system reliability evaluation," IEEE Trans. on Power Systems, vol. 13, no. 2, pp. 655-660, May 1998.

[16] J. A. Momoh, Y. V. Makarov, W. Mittelstadt, "A framework of voltage stability assessment in power system reliability analysis," IEEE Trans. on Power Systems, vol. 14, no. 2, pp. 484-491, May 1999.

[17] H. Wan, J. D. McCalley, V. Vittal, "Risk based voltage security assessment," IEEE Trans. on Power Systems, vol. 15, no. 4, pp. 1247-1254, Nov. 2000.

[18] A. B. Rodrigues, R. B. Prada, M. D. G. da Silva, "Voltage stability probabilistic assessment in composite systems: modeling unsolvability and controllability loss," IEEE Trans. on Power Systems, vol. 25, no. 3, pp. 1575-1588, Aug. 2010.

[19] N. Hatziargyriou (Chair) et al. "Contribution to Bulk System Control and Stability by Distributed Energy Resources connected at Distribution Network," IEEE/PES Power System Stability Subcommittee, Tech. Rep. PES-TR22, Jan 2017 